\title{
What, When, and How of Biomarker Testing in Non-Small Cell Lung Cancer
}

\author{
Presented by Gregory L. Riely, MD
}

\begin{abstract}
Biomarker testing is recommended for all patients diagnosed with non-small cell lung cancer. At a minimum, testing should include the mutations/fusions EGFR, ALK, ROS1, and the protein programmed death ligand-1 (PD-L1), because FDA-approved therapies are available for these alterations. Other actionable molecular findings include RET rearrangements, BRAF ${ }^{\mathrm{V} 600 \mathrm{E}}$ mutations, and MET exon 14 alterations. If adequate testing was not performed at treatment initiation, molecular testing should be performed before administration of subsequent lines of therapy. In patients with EGFR-mutant lung cancer, when resistance develops, physicians should seek to identify the T790M mutation using plasma and tissue assays, because osimertinib therapy is available for this mutation.
\end{abstract}

J Natl Compr Canc Netw 2017;15(5.5):686-688

Biomarker testing for patients with non-small cell lung cancer [NSCLC] is critically important, but it can be complicated," explained Gregory L. Riely, MD, Vice Chair, Clinical Research, Department of Medicine, Memorial Sloan Kettering Cancer Center, and a member of the NCCN NSCLC Panel. "Optimally, it should be done at diagnosis; it if it can't be done then, testing before giving second-line [or any subsequent line] therapy has value," Dr. Riely said.

Multiplexed testing (ie, putting several test results together) offers the best opportunity to learn about relevant markers. It is especially important to conduct biomarker testing (again) in the setting of resistance to initial therapy for patients with epidermal growth factor receptor (EGFR)-mutant NSCLC, he emphasized.

The 2017 NCCN Clinical Practice Guidelines in Oncology (NCCN Guidelines) for NSCLC recommend biomarker testing for all patients who present with stage IV disease. Molecular testing should be capable of identifying EGFR mutation, anaplastic lymphoma kinase $(A L K)$ gene rearrangements, and, for the first time, ROS1 and programmed death ligand-1 (PD-L1)

Presented by Gregory L. Riely, MD, Department of Medicine, Memorial Sloan Kettering Cancer Center, New York, New York.

Dr. Riely has disclosed that he has no financial interests, arrangements, affiliations, or commercial interests with the manufacturers of any products discussed in this article or their competitors.

Correspondence: Gregory L. Riely, MD, Memorial Sloan Kettering

Cancer Center, 300 East 66th Street, New York, NY 10065.

E-mail: rielyg@mskcc.org expression. The NCCN Guidelines state that testing should be conducted as part of broad molecular profiling.

"Molecular analysis and PD-L1 testing show you which direction to take" for first-line therapy, he continued.

In the absence of an identified mutated/rearranged driver oncogene, for patients with PD-L1 expression $<50 \%$, initial platinum-based therapy is recommended; this applies to approximately $45 \%$ of patients. If PDL1 expression is $>50 \%$, pembrolizumab should be given; this will apply to approximately $30 \%$ of patients (Figure $1)$. Molecular testing will indicate targeted therapy in approximately $25 \%$ of patients with NSCLC.

"We need to get all this testing done on a small biopsy. Get the most out of the tissue you have, and this includes PD-L1 testing," Dr. Riely told the audience.

Biomarkers are DNA-based (detected through sequencing and fluorescence in situ hybridization [FISH]) and protein-based (detected through immunohistochemistry [IHC]).

\section{PD-L1 Testing}

A variety of immune checkpoint inhibitors have become available, including those that target CTLA-4, PD-1, and PD-L1.

"First-line use of pembrolizumab has dramatically changed the treatment of metastatic NSCLC," Dr. Riely said. 


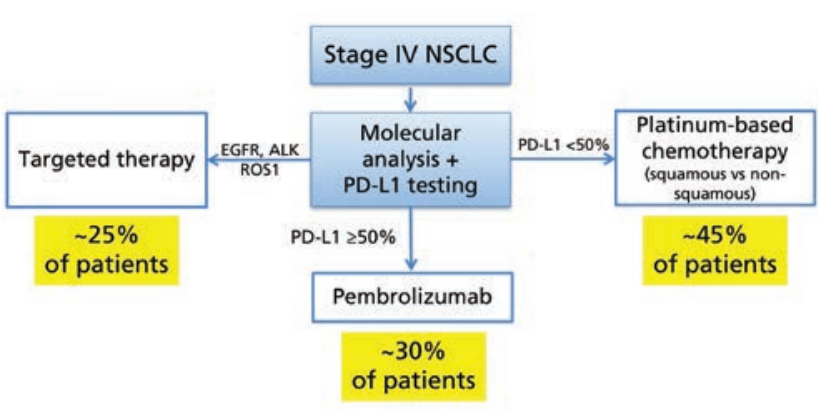

Figure 1. Approach to first-line treatment of patients with advanced non-small cell lung cancer (NSCLC).

PD-L1 expression of at least $50 \%$ on the tumor is required for pembrolizumab to be used as initial treatment. Studies have shown that patients with stage IV NSCLC with PD-L1 $>50 \%$ have significantly superior overall survival on pembrolizumab versus chemotherapy $(P=.005){ }^{1}$

"There is no difference in survival when a lower PD-L1 cutoff of $5 \%$ is used," Dr. Riely said. "With the data available, the threshold of $50 \%$ is the right cutoff, and this is key."

"Tests for PD-L1 expression are all over the map. These tests use a variety of antibodies. The test for pembrolizumab is the most widely used, because the label guides us to use this to make first-line treatment decisions," he continued.

Four different assays are available, each one linked to a different available immunotherapy. A study that examined the same tumors stained with 4 different PD-L1 assays found general concordance among 3 of them (ie, 28-8 for nivolumab, 22C3 for pembrolizumab, SP263 for durvalumab), with SP142 (the assay linked to atezolizumab) an outlier. ${ }^{2}$

"PD-L1 testing is generally concordant, with one exception," he said.

\section{Timing of Biopsy for Testing}

One issue is the timing of biopsy. Should a biopsy be obtained just before starting therapy, and should a repeat biopsy be performed later?

Dr. Riely said studies show that PD-L1 expression is relatively stable when tested at 2 different time points. "This suggests that testing the archival sample may be adequate," he said. "There is no clear benefit for repeat biopsy for assessment of PD-L1, unless the prior sample is exhausted," he said.
"In summary, PD-L1 testing should be done on all patients with newly diagnosed metastatic NSCLC. This gives you the opportunity to give first-line immunotherapy for patients with PD-L1 expression $>50 \%$," he emphasized.

\section{Molecular Testing}

NSCLC is characterized by several altered driver oncogenes, including KRAS (21\%), EGFR (17\%), HER2 (2\%), RET fusions (2\%), ROS1 fusions (2\%), ALK fusions (2\%), BRAF ${ }^{\mathrm{V} 600 \mathrm{E}}(1 \%)$, and MET exon 14 alterations (2\%). Other genotypes account for $50 \%$ of NSCLC. There is no available therapy to target KRAS, Dr. Riely said.

EGFR is now recognized as a targetable mutation in NSCLC. Early studies of erlotinib showed a low response rate, but 1 in 10 patients had a dramatic response to the drug. When patients with a high response were studied more closely, they showed EGFR mutations. In 2004, EGFR mutations were identified and associated with response to both erlotinib and gefitinib, Dr. Riely explained.

"Numerous prospective trials have shown that EGFR tyrosine kinase inhibitors [erlotinib, gefitinib, afatinib] are more effective than platinum doublets in patients with EGFR-mutant NSCLC," he said.

Other actionable targetable mutations in NSCLC include ALK fusions (treatable with crizotinib, ceritinib, and alectinib), BRAF ${ }^{\mathrm{V} 600 \mathrm{E}}$ (treatable with the combination of dabrafenib and trametinib), ROS1 fusions (treatable with crizotinib), RET fusions (treatable with cabozantinib), and MET exon 14 (treatable with crizotinib).

"For molecularly targeted therapies, the line of therapy may not matter," Dr. Riely noted. In one study, progression-free survival curves were superimposed for first- and second-line treatment. ${ }^{3}$

These molecular abnormalities can be difficult to detect. The NCCN Guidelines recommend molecular testing as part of broad molecular profiling. Testing for EGFR, ALK, and ROS1 are category 1 recommendations. PD-L1 testing should be performed for all patients with NSCLC.

IHC can be used to detect PD-L1, ALK, ROS 1, and, rarely, EGFR mutations. FISH can be used to detect ALK, ROS, and RET. DNA sequencing is useful for EGFR, BRAF, and MET exon 14 mutations. 
Riely

"Biomarker testing needs to be customized at each institution," Dr. Riely said. "There is no single answer for all institutions. The goal is to return results to the treating oncologist within 10 days. Often, you need a combination of techniques to achieve comprehensive evaluation in a timely manner," he explained.

Despite initial response rates, patients will eventually develop resistance to EGFR tyrosine kinase inhibitors (TKIs). More than $60 \%$ of EGFR-mutant patients will have a new T790M mutation at the time of resistance. This mutation cannot be targeted with a first- and second-generation EGFR TKI, but osimertinib, a third-generation EGFR TKI, is effective against the T790M mutation in patients with acquired resistance to gefitinib, erlotinib, and afatinib.
When patients with EGFR-mutated NSCLC experience disease progression on first- or secondgeneration EGFR TKI treatment, they should be tested for plasma T790M, Dr. Riely said. If the test is positive, treatment with osimertinib is recommended. If the plasma test is not positive, tissue testing should then be performed by obtaining a biopsy. Patients who are T790M-negative should be treated with chemotherapy.

In summary, EGFR, ALK, and ROS1 are examples of targetable oncogenic drivers in lung cancer. Routine testing for these oncogenes is standard for patients with NSCLC. Patients with EGFR-mutant NSCLC should have repeat molecular profiling performed upon resistance to firstline EGFR TKI therapy.

\section{References}

1. Reck M, Rodriguez-Abreu D, Robinson AG, et al. Pembrolizumab versus chemotherapy for PD-L1-positive non-small-cell lung cancer. N Engl J Med 2016;375:1823-1833.

2. Rimm DL, Han G, Taube JM, et al. A prospective, multi-institutional, pathologist-based assessment of 4 immunohistochemistry assays for PDL1 expression in non-small cell lung cancer [published online ahead of print March 9, 2017]. JAMA Oncol, doi: 10.1001/jamaoncol.2017.0013.

3. Rosell R, Moran T, Queralt C, et al. Screening for epidermal growth factor receptor mutations in lung cancer. N Engl J Med 2009;361:958-967.

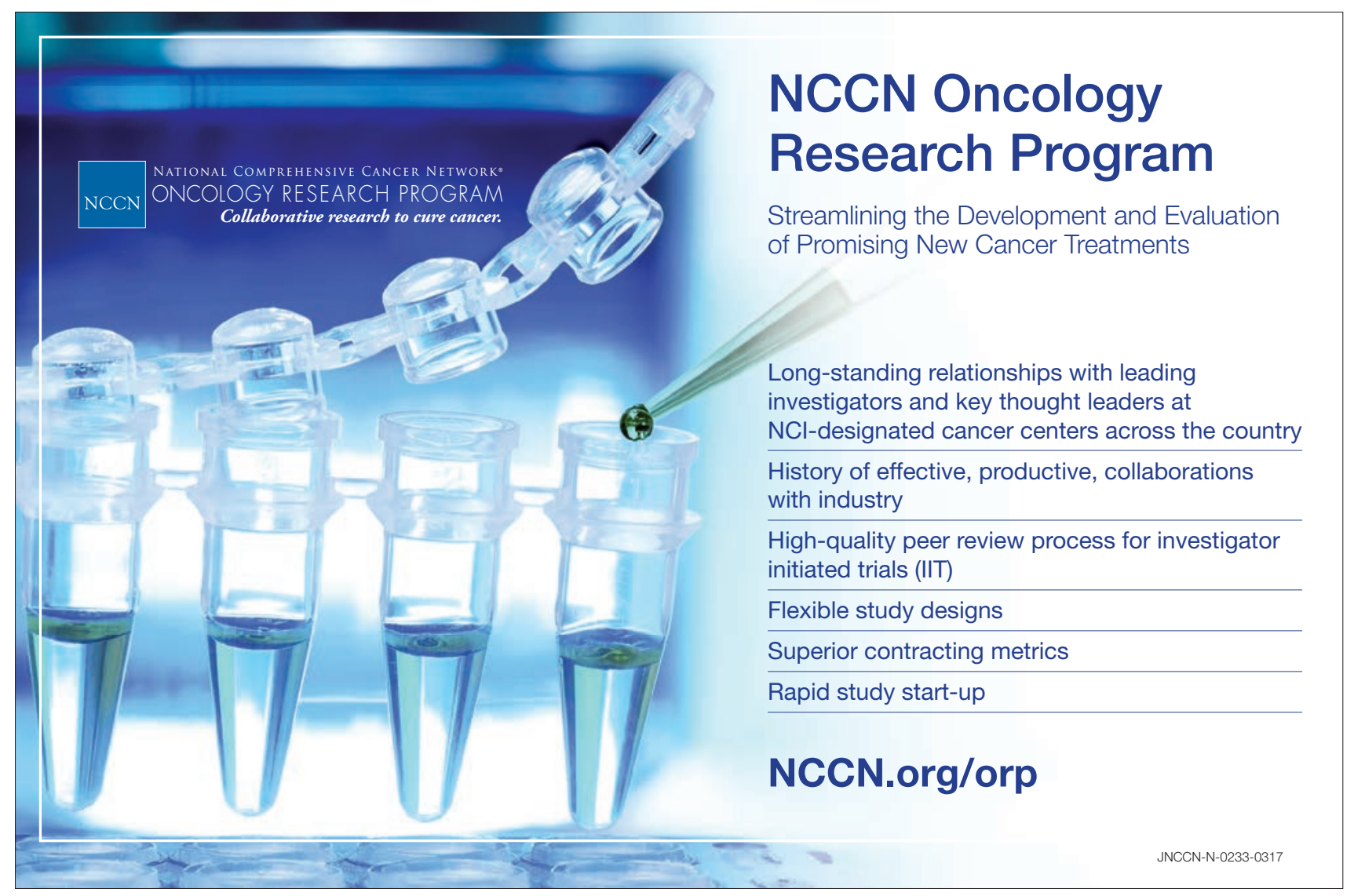

